\title{
Oral health and respiratory diseases
}

\author{
Is there an association between oral health and pneumonia or other \\ respiratory diseases?
}

\author{
Azarpazhooh A, Leake JL. \\ Systematic review of the association between respiratory diseases \\ and oral health. J Periodontol 2006; 77:1465-482.
}

Data sources Relevant studies were sourced using Ovid Medline (In-Process and Other Non-Indexed Citations, Daily Update and OLDMedline); Cumulative Index to Nursing and Allied Health Literature; Evidence Based Medicine of Cochrane Central Register of Controlled Trials; Cochrane Database of Systematic Reviews; Database of Abstracts of Reviews of Effects; Embase; Health and Psychosocial Instruments; HealthSTAR; International Pharmaceutical Abstracts; PubMed; and Google Scholar.

Study Selection Randomised controlled clinical trials and longitudinal, cohort, case-control and epidemiological studies were selected. Searches were limited to English language and human studies.

Data extraction and synthesis Data were extracted independently, evidence tables constructed and a qualitative summary made of the data.

Results Nineteen studies met the inclusion criteria. In five studies, the presence of cariogenic and periodontal pathogens, dental decay, and poor oral hygiene were identified as potential risk factors for pneumonia. A weak association between periodontal disease and chronic obstructive pulmonary disease (COPD) was identified in four poor to fair studies. Ten studies provided evidence that interventions aiming to improve oral health reduced the progression or occurrence of pneumonia.

Conclusions First, there is fair evidence (II-2, grade B recommendation) of an association of pneumonia with oral health [odds ratio (OR), 1.2-9.6 depending on oral health indicators]. Second, there is poor evidence of a weak association $(\mathrm{OR}<2.0)$ between COPD and oral health (II-2/3, grade $\mathrm{C}$ recommendation). Third, there is good evidence (I, grade A recommendation) that improved oral hygiene and frequent professional oral healthcare reduces the progression or occurrence of respiratory diseases in high-risk elderly adults living in nursing homes and especially those in intensive care units (number needed to treat, 2-16; relative risk reduction, 34-83\%).

\section{Commentary}

As readers of this journal are aware, there is currently much interest in the relationship between oral and general health. As they will also recognise, demonstrating that oral health is in some way a cause of systemic disease is a challenge.

The rationale for this comprehensive systematic review was that several observational studies report an association between poor oral health and respiratory diseases (COPD and community- and hospital-acquired pneumonia). In addition, clinical trials have begun to test the effect of improving oral health on pneumonia. The consistency of the findings and the quality of the research is not clear, how-

Address for correspondence Dr Amir Azarpazhooh, Department of Community Dentistry, Faculty of Dentistry, University of Toronto, Toronto, Ontario, Canada. E-mail: amir.azarpazhooh@utoronto.ca ever. These respiratory diseases are significant causes of death and morbidity and are hugely expensive to manage. Therefore, preconditions for conducting a systematic review were well met, ie, clinical uncertainty in an important health issue.

The methods used in the review were based on a Canadian Workshop model and were rigorous. A detailed search of multiple databases was conducted, although the search was limited to Englishlanguage publications and to local journal holdings. Resources for translation are not always easy to secure but it would have been interesting to know how many publications were excluded on this basis. As it is, the risk of publication bias (which favours the reporting of trials with positive outcomes) cannot be assessed. The criteria for eligibility of studies and an explanation of the methods for critical appraisal were not explicit, limiting assessment of these aspects.

One critical issue in appraising the original studies for this review was the security of diagnosis of the respiratory and oral diseases. Clearly, without confidence that diagnosis was appropriate, it would be difficult to have any security in the findings of the studies included or of the systematic review. Explicit criteria are set out by Azarpazhooh and Leake for the clinical trials and these diagnostic criteria are appropriate and in routine clinical use. For the observational studies, however, explicit criteria are not reported, although this might result from a deficiency of the original studies. As a result, understanding the relationship between oral health and respiratory diseases from observational studies is problematic.

What is clearer is that oral health interventions can reduce the incidence or progression of pneumonia acquired in long-term institutionalised-care facilities or hospitals. Further research will be needed to identify the interventions with the best benefit/ harm ratios and, in particular, those that can be routinely applied within the context of the (often complex) medical care that individuals will already be receiving. Collaborations between oral health and medical researchers should therefore be considered mandatory. In view of the potential impact of such simple interventions on the health of individuals, appropriate investigations should be undertaken with urgency.

\section{lan Needleman}

International Centre for Evidence-Based Oral Health, Unit of

Periodontology, University College London Eastman Dental

Institute, London UK

Nicholas Hirsch

Neuromedical Intensive Care Unit, National Hospital for Neurology and Neurosurgery, University College London Hospital NHS Foundation Trust, London, UK

\section{Conflict of interest}

Drs Needleman and Hirsch are currently conducting a randomised controlled trial investigating the impact of oral health on ventilator-associated pneumonia. Evidence-Based Dentistry (2007) 8, 116. doi:10.1038/sj.ebd.6400532 